\title{
Nano-formulations composed of cell membrane-specific cellular lipid extracts derived from target cells: physicochemical characterization and in vitro evaluation using cellular models of breast carcinoma
}

\author{
Hanan M. Alharbi and Robert B. Campbell
}

\begin{abstract}
Highly selective drug targeting is an important goal in the development of cancer nanotechnologies. In an effort to improve tumor targeting a method was developed to formulate cell membrane lipid-extracted nanoliposomes (CLENs). The main ingredients were extracted directly from the membrane of cancer cells. For this study we used three different breast cancer cell lines (4 T1, BT-20, and SK-BR-3). As controls for the normal breast and cancer tissue environments we employed the normal breast fibroblast (CRL-2089) and ovarian cancer (SK-OV-3) cell lines, respectively. We evaluated physicochemical properties, efficiency of drug loading, cellular uptake, and cytotoxicity. The mean diameter and zeta potential values for the 5 different CLENs were $202 \pm 38 \mathrm{~nm}$ and $-15 \pm 3.8 \mathrm{mv}$, respectively. Doxorubicin hydrochloride ( 5 mol\%) increased the size of 4 T1-CLENs from $158 \pm 2 \mathrm{~nm}$ to $212 \pm 59 \mathrm{~nm}$, with no significant change in the negativelycharged surface potential. Percent of drug loaded ranged from 40 to $93 \%$, varying according to the ratio of lipid extract to conventional components employed. The additional inclusion of cholesterol and DPPE-PEG 5000 increased drug loading in CLENs, similar to Doxil preparations. The most promising cellular uptake and cytotoxicity profiles were observed when the lipid ingredients were derived from the eventual target cell. Given the ability of CLENs to better recognize target cells compared to nanosystems consisting of non-specific lipid extracts or conventional liposome ingredients alone, CLENs has demonstrated early promise as a nano-delivery system for cancer treatment.
\end{abstract}

Keywords: Breast cancer, Cell lines, Cholesterol, Liposomes, Drug delivery systems

\section{Background}

An important goal of chemotherapy is to eradicate the tumor mass while causing minimal side effects to the patient. Liposomes have been employed to help achieve this goal. The vesicle type has improved tumor targeting and when optimised can favorably alter the pharmacokinetic and biodistribution profile of the drug. (Campbell et al., 2002; Deshpande et al., 2013; Torchilin, 2005) Although effective, issues involving limited interstitial drug transport, off-target drug effects and formulation instability still remain. (Deshpande et al., 2013; Torchilin, 2005; Sharma et al., 2006).

\footnotetext{
* Correspondence: robert.campbell@mcphs.edu

Department of Pharmaceutical Sciences, MCPHS University, 19 Foster Street, Worcester, MA 01608, USA
}

A current area of research investigation involves the development of nanomedicines capable of recognizing, and selectively targeting, exploitable tumor features for cancer therapy. (Campbell et al., 2002; Torchilin, 2005; Sharma et al., 2006; Campbell, 2006; Sadzuka et al., 2003) A few advances in the field of nanoliposome development include the inclusion of poly-ethylene glycol (PEG)-PE in nano-size drug delivery systems (i.e., liposomes, micelles). (Dan, 2002) The liposome formulation product Doxil contains PEG, and as a result can successfully exploit the relatively large tumor vascular pore openings owing to extended circulation properties afforded by PEG. (Sadzuka et al., 2003; Eloy et al., 2014; Hatakeyama et al., 2013; Jain \& Jain, 2008; Ryan et al., 2008; Vemuri \& Rhodes, 1995; Verma et al., 2008) PEG has versatile functions. For 
example, its inclusion in cationic liposomes permits preferential tumor targeting, relatively longer circulation of cationic liposomes while significantly decreasing uptake by vessels in healthy tissues. (Campbell et al., 2002; Campbell, 2006) Efforts to both gain access to and exploit the differential expression of various receptors in tumors for treatment when compared to normal tissues has improved with advances in liposome technology. (Torchilin, 2005; Sharma et al., 2006; Lu \& Low, 2002) Such examples include the significant folate receptor-mediated uptake in athymic mice-bearing tumors overexpressing folate receptor-positive cells, compared to non-folate receptor-labeled systems for which reduced uptake was observed. (Mathias et al., 1998)

Regardless of the specific variety of nanomedicine employed most drug delivery systems are prepared from natural and/or synthetic lipid ingredients. Moreover, the ratio of the ingredients employed rarely resemble the composition profile of the target cell membrane. In an effort to achieve more selective targeting we designed, prepared and evaluated the use of cell membrane lipid-extracted nanoliposomes (CLENs) in vitro. The process involved the extraction of cellular lipid material from intended target cells, and the use of extracted lipid material with (and without) conventional ingredients for formulation and in vitro experimentation. Moreover, a single organ tissue environment was the primary focus of this investigation. The tissue model was additionally selected to represent an organ tissue environment for which the nano drug platform is being developed. For this reason, the cellular lipid material used to prepare CLENs was derived from breast tissue, except for when negative controls were employed.

The composition profile of extracted lipid components of CLENs more closely resemble the target cell membrane compared to non-specific preparations in terms of composition. For this reason, we investigated whether CLENs prepared from lipid extracts derived from target cells will associate with the intended target cells to a greater extent when compared to CLENs prepared from non-specific lipid extracts, and/or conventional nanoliposomal preparations.

\section{Methods}

\section{Materials}

The lipids 1, 2-dioleoyl-Sn-glycero-3-[phospho-rac-(1-glycerol)] (DOPG), 1,2-dioleoyl-sn-glycero-3-phosphocholine (DOPC), Cholesterol (Chol), 1,2-dipalmitoryl-sn-glycero-3[phospho-ethanolamine (Polyethylene glycol)]-5000 (DPPE -PEG 5000 ), 1,2-dipalmitoyl-sn-glycero-3-phosphoethanolami ne-N- (lissamine rhodamine B sulfonyl) (rhodamine -DPPE$_{\text {) }}$ ), were purchased from Avanti Polar Lipids (Alabaster, AL). Sulforhodamine B (SRB) and Doxorubicin hydrochloride (98\% HPLC) were purchased from Sigma-Aldrich (St Louis,
MO). All chemicals and solvents used in this study were of analytical grade and obtained from Fisher Scientific (Pittsburgh, PA).

\section{Cell culture}

Human breast cancer cell lines BT-20 (HTB-19), SKBR3 (HTB-30), murine mammary cell line 4 T1 (CRL-2539), normal mammary fibroblast cell line CCD-1069SK (CRL-2089), and ovarian cancer cell line SK-OV-3 (HTB-77) were obtained from ATCC (American Type Culture Collection, Manassas, VA). All cell line cultures were grown in a humidified atmosphere of $5 \% \mathrm{CO}_{2}$ at $37{ }^{\circ} \mathrm{C}$.

\section{Cellular lipid extraction}

The method used to extract the cellular lipid material was modified from the original Bligh and Dyer method established in 1959. (Dabbas et al., 2008) The standard gravimetric quantification method involves the use of three different solvents. In brief, when the cells reached approximately $90 \%$ confluence, they were trypsinized and collected. The pellet of cells was diluted with 1X PBS (phosphate buffer saline). The average number of cells in a pellet determined the proportion of solvents used according to a modified chart (Table 1). The three solvents were added step-wise as follows: 1:2 chloroform-methanol mixture, chloroform, and distilled water, vortexed intermittently between solvent additions. The final mixture was centrifuged at $1000 \mathrm{rpm}$ at $4{ }^{\circ} \mathrm{C}$ for 5 min to obtain a two-phased system; the bottom lipid layer was aspirated and transferred into a glass tube and refrigerated until a sufficient volume was obtained.

\section{Lyophilization method}

The extracted lipids were dehydrated to form a film using a rotary evaporator system, and a heating bath (Buchi B-491) (Flawil, Switzerland) with temperatures maintained at $40-50{ }^{\circ} \mathrm{C}$. Sucrose $(0.2 \mathrm{M})$ was added to serve as a cryoprotectant during the process of lyophilization using a FreeZone Freeze Dry system (Labconco, Kansas City, MO). The lipid powder was weighed and subsequently dissolved in $1 \mathrm{~mL}$ of chloroform. All lipid chloroform stocks used to prepare CLENs were stored at $-80{ }^{\circ} \mathrm{C}$. Prior to using the lipid extracts for preparation of CLENs batches of the freeze dried lipid extract were evaluated by LCMS. All lipid components were identified, and the relative abundance and molecular weights for each lipid extract type was determined, resulting in average molecular weights. The final concentrations were subsequently determined for the lipid extract stocks and 
Table 1 Modified chart of the Dyer and Bligh lipid extraction method

\begin{tabular}{llllll}
\hline Cell Number (millions) & $(5-9.9)$ & $(10-24.9)$ & $(25-49.9)$ & $(50-74.9)$ & $(75-99.9)$ \\
\hline $1: 2 \mathrm{CHCl}_{3}: \mathrm{MeOH}$ & 0.750 & 1.9 & 3.75 & 5.7 & 7.5 \\
$\mathrm{CHCl}_{3}$ & 0.250 & 0.625 & 1.25 & 1.875 & 2.5 \\
$\mathrm{dH}_{2} \mathrm{O}$ & 0.250 & 0.625 & 1.25 & 1.875 & 2.5 \\
Total Volume & 1.45 & 3.65 & 7.25 & 10.95 & 14.50 \\
\hline
\end{tabular}

later used to prepare CLENs in absence and presence of drug agents.

\section{CLENs and conventional liposome preparations}

The CLENs used in this study were generally derived from breast cancer cell lines. We named the CLENs by the cell line from which the lipid material was derived (i.e., 4 T1 CLENs, BT-20 CLENs etc.). The composition of the nanoliposome formulation employed

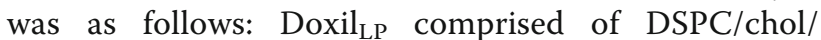
PEG-5000 (50/45/5). 4 T1 CLENs were employed with the inclusion of chol $(0,10,25$, or $50 \mathrm{~mol} \%)$ and DPPE-PEG ${ }_{5000}(0,2,5$, or $10 \mathrm{~mol} \%)$. When necessary, rhodamine-DPPE label was included in CLENs at the ratio of $1 \mathrm{~mol} \%$. CLENs were prepared by thin film hydration method as previously reported (Campbell et al., 2002; Dandamudi \& Campbell, 2007; Kalra \& Campbell, 2006; DeSantis et al., 2014). Particle size and zeta $(\zeta)$ potential were determined following five minutes of sonication using a 90 Plus Particle/Zeta Potential Analyzer (Brookhaven Instruments, Holtsville, NY).

\section{Drug incorporation efficiency}

Doxorubicin hydrochloride (5 mol\%) was loaded in conventional and 4 T1 CLENs. To determine drug incorporation efficiency, the two fractions (incorporated and un-incorporated free drug) were separated. First, a required volume was removed from each preparation type and stored at $4{ }^{\circ} \mathrm{C}$. Next, each preparation was centrifuged using an ultra-centrifugation system at $13,000 \mathrm{rpm}$ for $15 \mathrm{~min}$, and a pre-determined volume was removed from the preparation and stored at $4{ }^{\circ} \mathrm{C}$. The centrifuged formulation was transferred to a Float-A-lyzer system with $1000 \mathrm{MW}$ semipermeable membrane (Fisher Scientific, Pittsburgh, PA) and placed in a beaker filled with $1 \mathrm{X}$ PBS at $4{ }^{\circ} \mathrm{C}$ overnight. The next day, a required volume from the dialyzed formulation was removed and used for analysis. The three samples (before centrifugation, after centrifugation, and after dialysis) were added to a 96-well plate to determine doxorubicin fluorescence intensity using a fluorescence microplate reader. The fluorescence intensity was measured at excitation and emission wavelengths of 540/20 $\mathrm{nm}$ and 590/20 nm, respectively. The percentage of doxorubicin incorporated was calculated using the following equation:

Percent of drug Incorporated

$=\frac{\text { Fluorescence intensity (after dialysis sample) }}{\text { Fluorescence intensity (before dialysis sample) }} \times 100$

\section{Cellular uptake studies}

Cells were seeded at $1 \times 10^{4} / \mathrm{mL}$ in a 48 -well plate and incubated at $37{ }^{\circ} \mathrm{C}$. Following $24 \mathrm{~h}$ of incubation, rhodamine labeled CLENs prepared from specific cell lines were added to the respective well at different concentrations. Following an additional $24 \mathrm{~h}$ of incubation the plates were washed with 1X PBS and analyzed using a fluorescence microplate reader (BioTek Instruments, Winooski, Vermont).

\section{Cytotoxicity of doxorubicin-loaded CLENs}

Sulforhodamine B (SRB) assay was used to determine percent viability of control based on the amount of basic proteins in viable cells following exposure to different CLENs. Cells were seeded at $1 \times 10^{4}$ per $\mathrm{ml}$ in a 48 -well plate. Columns of cells in the 48 well plate were exposed to either doxil, free doxorubicin, SKBR-3, BT-20 or 4 T1 CLENs-loaded with doxorubicin (5 mol\%). Following a $24 \mathrm{~h}$ incubation period at $37{ }^{\circ} \mathrm{C}$, cells were treated with various concentrations of different types of CLENs loaded with doxorubicin $\mathrm{Hcl}$. The following day, SRB assay was utilized to determine the percent of cell viability. Briefly, plates were washed twice with $1 \mathrm{X}$ PBS and the cells were fixed with 50\% wt/vol TCA (Trichloroacetic acid) and stored at $4{ }^{\circ} \mathrm{C}$ for $1 \mathrm{~h}$. Next, plates were washed five times by distilled deionized water, stained by $0.4 \% w / v$ of SRB dye, and placed in the dark for $30 \mathrm{~min}$. Excess dye was washed minimum of four times with $1 \% \mathrm{v} / \mathrm{v}$ acetic acid and left to dry completely. Finally, $1 \mathrm{~mL}$ of $1 \mathrm{X}$ PBS was added to each well and the plate was analyzed using a fluorescence microplate reader. The fluorescence intensity was measured at excitation wavelength of $540 / 20 \mathrm{~nm}$ and emission wavelength of 590/20 nm using FLX 800 Fluorescence Microplate Reader (Bio-tech Instruments, Winooski, VT).

\section{Cellular toxicity of CLENs}

To determine the level of cellular toxicity of CLENs compared to controls the Sulforhodamine B assay was performed as reported elsewhere (Dabbas et al., 2008; 
Dandamudi \& Campbell, 2007; Kalra \& Campbell, 2006), and as briefly described under the section cytotoxicity of doxorubicin-loaded CLENs.

\section{Statistics}

Statistical analysis was performed using ANOVA (analysis of variance) followed by Turkey's multiple comparison procedure as post- hoc, and two-tailed Student's t-test using Sigma Plot for Windows (Systat Software, San Jose, CA). Results are presented as mean \pm SD, $n=6$. $P<0.05$ and $P<0.001$ were considered statistically significant and denoted as $*$ and ${ }^{* *}$, respectively.

\section{Results}

\section{Characterization of CLENs}

The physicochemical properties such as particle size and surface charge potential are important to assess the quality of the preparation, including drug incorporation, stability and drug release characteristics. Particle size and zeta $(\zeta)$ potential of the different CLENs were determined using the PALS zeta potential analyzer. The unimodal particle size distribution ranged between 150 and $263 \mathrm{~nm}$, with an increase in size observed for preparations including cholesterol and DPPE-PEG-5000. All preparations exhibited negative zeta potential values ranging between -11 to $-21 \mathrm{mV}$ (Table 2).

\section{Incorporation efficiency of doxorubicin in 4 T1-CLENs}

The percent of drug incorporated in a drug carrier molecule is an important step in formulation development. For this reason, we evaluated the incorporation efficiency for doxorubicin in 4 T1-CLENs (Table 3). The inclusion of $25 \mathrm{~mol} \%$ cholesterol, and DPPE-PEG-5000 (2 or $5 \mathrm{~mol} \%$ ) in 4 T1-CLENs demonstrated the best results. Drug incorporation was similar to the incorporation of doxorubicin in Doxil (Table 3). The incorporation of the drug increased the size of 4 T1-CLENs, with no observable effect on the values for zeta potential.

\section{In vitro cellular toxicity profile of CLENs}

The SRB assay was performed for different preparations of CLENs and compared to the relative effects of conventional liposomes. The toxicity profile was determined

\begin{tabular}{|c|c|c|c|}
\hline \# & Nanoliposomal Preparations & Particle Size $(\mathrm{nm})$ & Zeta potential $(\mathrm{mV})$ \\
\hline 1 & Doxil LP & $150 \pm 2.0$ & $-23 \pm 0.9$ \\
\hline 2 & 4 T1 CLENs & $158 \pm 1.9$ & $-17 \pm 2.5$ \\
\hline 3 & BT-20 CLENs & $263 \pm 1.4$ & $-21 \pm 3.5$ \\
\hline 4 & SK-BR-3 CLENS & $187 \pm 2.1$ & $-13 \pm 1.1$ \\
\hline 5 & CRL-2089 CLENs & $203 \pm 1.9$ & $-15 \pm 1.3$ \\
\hline 6 & SK-OV-3 CLENS & $200 \pm 2.2$ & $-11 \pm 2.3$ \\
\hline
\end{tabular}

for four different cell lines: three mammary epithelial (4 T1, BT-20, SK-BR-3), and one mammary fibroblast (CRL-2089). Each cell line variety was exposed to five different types of CLENs. A toxicity profile for each preparation is shown in Fig. 1. All CLENs demonstrated a relatively non-toxic effect against cellular growth within the concentration range evaluated. In comparison to the untreated control minimal toxicity was observed for CLENs. CLENs demonstrated similar toxicity profiles

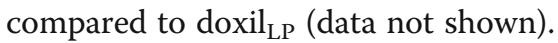

\section{Cellular uptake of CLENs}

Cellular uptake studies were performed to determine the extent to which CLENs were taken up by various breast cancer cell lines. Figure 2 showed significant cellular uptake of CLENs when 4 T1 cells were exposed to 4 T1 CLENs, compared to other CLEN varieties. Similar results were observed for when the BT-20 cell line was exposed to BT-20 CLENs (Fig. 3). Minimal uptake was observed for when SK-OV-3 (the negative control) cells were exposed to both $4 \mathrm{~T} 1$ and BT-20 CLENs (Figs. 2 and 3). Four different preparations of 4 T1-CLENs all containing $25 \mathrm{~mol} \%$ of cholesterol and different ratios of DPPE-PEG-5000 were evaluated for cell uptake against the $4 \mathrm{~T} 1$ cell line (Fig. 4). 4 T1-CLENs containing $5 \mathrm{~mol} \%$ of DPPE-PEG-5000 demonstrated significant uptake compared to the other PEG-containing preparations (Fig. 4). In the next study, we used the optimized ratio of PEG-5000 and compared the CLENs type to a nano-system with a lipid composition resembling Doxil (referred to here as Doxil ${ }_{\text {LP }}$ (Doxil lipid preparation without doxorubicin)). The inclusion of DPPE-PEG-5000 (5 mol\%) significantly enhanced the cellular uptake of CLENs when compared to Doxil LP (Fig. 5).

\section{Cytotoxicity studies of doxorubicin-loaded CLENs}

Enhanced cellular uptake and selective drug targeting are important features of a drug delivery system, but how each translates overall to therapy is critical to success. For this reason, we next evaluated cytotoxicity of three different doxorubicin-loaded CLENs for 4 T1, BT-20 and SKBR-3 variety, doxil, and free doxorubicin $(0.5 \mu \mathrm{mol} / \mathrm{ml})$ against the growth of $4 \mathrm{~T} 1$ cells in vitro. The results showed a significant decrease in the growth of $4 \mathrm{~T} 1$ cells following exposure to $4 \mathrm{~T} 1$ doxorubicin-loaded CLENs compared to untreated cells $(P<0.001)$. The percent of viable cells was 50 and $46 \%$ for concentrations of 100 and $150 \mathrm{nmol} / \mathrm{mL}$, respectively (Fig. 6). We observed no significant difference in cytotoxicity among $4 \mathrm{~T} 1$ doxorubicin-loaded CLENs, doxil, and free doxorubicin. (Fig. 6). Interestingly, the non-specific SKBR-3 CLENs, for which similar amounts of drug agent was incorporated, was significantly less effective compared to 4 T1 doxorubicin-loaded CLENs, doxil, and free doxorubicin (data not shown). 
Table 3 Characterization and evaluation of 4 T1 CLENs loading efficiency

\begin{tabular}{|c|c|c|c|c|c|}
\hline \# & Nanoliposomes & mol\% & Particle Size (nm) & Z-Potential (mV) & Dox Incorporation Efficiency \% \\
\hline 1 & $4 \mathrm{~T} 1 / \mathrm{chol}$ & $100 / 0$ & $131 \pm 6.5$ & $-14 \pm 0.4$ & $63 \pm 1.3$ \\
\hline 2 & $4 \mathrm{~T} 1 / \mathrm{chol}$ & $90 / 10$ & $184 \pm 0.4$ & $-20 \pm 1.2$ & $64 \pm 1.9$ \\
\hline 3 & $4 \mathrm{~T} 1 /$ chol & $75 / 25$ & $239 \pm 4.3$ & $-18 \pm 2.50$ & $86 \pm 0.9$ \\
\hline 4 & $4 \mathrm{~T} 1 / \mathrm{chol}$ & $50 / 50$ & $282 \pm 0.3$ & $-20 \pm 2.50$ & $44 \pm 0.9$ \\
\hline 5 & $4 \mathrm{~T} 1 / \mathrm{chol} / \mathrm{PEG}_{-5000}$ & $73 / 25 / 2$ & $180 \pm 5.2$ & $-10 \pm 1.2$ & $81 \pm 1.5$ \\
\hline 6 & 4 T1/ chol /PEG-5000 & $70 / 25 / 5$ & $179 \pm 0.2$ & $-13 \pm 1.5$ & $93 \pm 0.9$ \\
\hline 7 & $4 \mathrm{~T} 1 /$ chol /PEG-5000 & $65 / 25 / 10$ & $290 \pm 0.4$ & $-10 \pm 1.7$ & $41 \pm 0.3$ \\
\hline 8 & Doxil $_{L P}$ & $45 / 50 / 5$ & $168 \pm 0.6$ & $-13 \pm 1.5$ & $95 \pm 1.1$ \\
\hline
\end{tabular}

\section{Discussion}

Breast cancer is a major cause of cancer death in women worldwide (Hassan et al., 2010; Jemal et al., 2011; Moulder \& Hortobagyi, 2008; Rivenbark et al., 2013). The disease is characterized by variant pathological features, disparate responses to therapeutics, and substantial differences in patient's long-term survival (Verma et al., 2008; Jemal et al., 2011; Rivenbark et al., 2013; Taylor et al., 1990). Targeted drug delivery systems (i.e., liposomes) can improve the pharmacokinetic and bio-distribution profile of commonly used drugs used for the treatment of disease (Campbell et al., 2002; Torchilin, 2005; Sharma et al., 2006; Campbell, 2006; Kalra \& Campbell, 2006; Allen et al., 1991). Our studies were performed to evaluate different nanoliposome formulations derived from natural lipid extracts from various breast cancer cell lines. Perhaps the lipid extracts could one day be optimized to target specific stages of tumor maturation and development. However, the general procedure employed for lipid extraction presented here was optimized for cell lines regardless of staging. Once the procedure was established no additional modifications were necessary (Fig. 7). CLENs contain a wider range of different lipid components with varied acyl chain lengths and degree of unsaturation compared to more conventional liposomal preparations. In CLENs the lipids are naturally employed in ratios unique to the target cell, and the fractional makeup of the many different lipid components appears to make them less recognizable by non-target cell populations. This is ideal when selective drug targeting if needed.

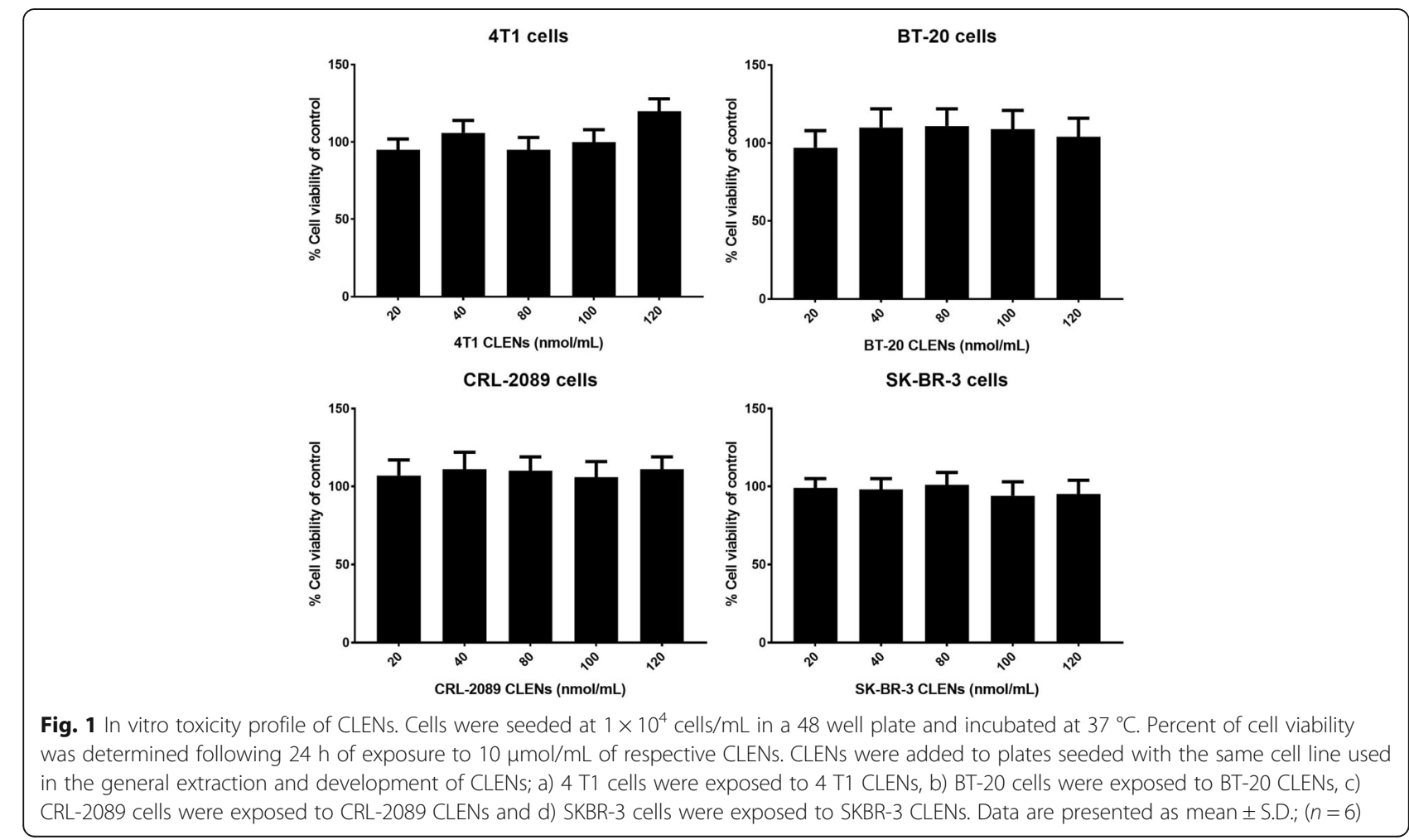




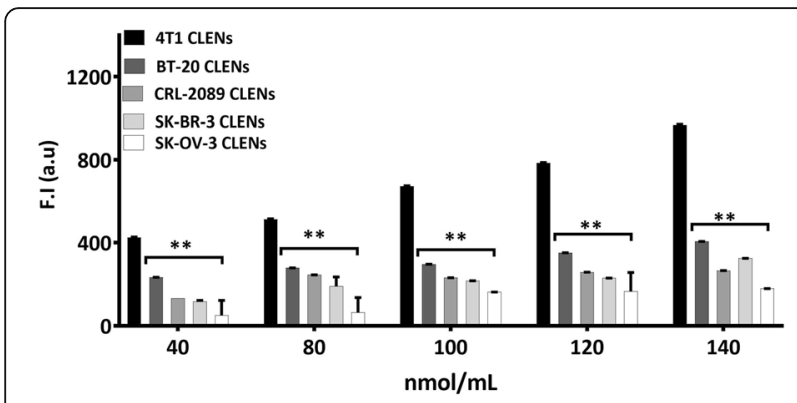

Fig. 24 T1 cellular uptake studies. An amount of $1 \times 10^{4} 4$ T1 cells were seeded in a 48 well plate. After $24 \mathrm{~h}$, cells were exposed to different concentration of fluorescently labeled CLENs and incubated with the cells for an additional $24 \mathrm{~h}$. The most significant cellular uptake was observed with $4 \mathrm{~T} 1$ cells compared to the other breast cells. The least amount of cellular uptake was observed with the ovarian cancer (negative control) cell line, SK-OV-3. Data are presented as mean \pm S.D.; $(n=6)$

Investigating the physicochemical characteristics of a drug carrier molecule is an important step to understanding formulation quality, stability, drug release rates and intracellular fate. The average particle size for CLENs was between 100 and $200 \mathrm{~nm}$, the ideal size range for I.V. administered formulations (Torchilin, 2005; Sharma et al., 2006; Campbell, 2006; Pasenkiewicz-Gierula et al., 2000). The zeta potential values for CLENs were negatively-charged, suggesting the vehicle is likely to accumulate in the tumor interstitial environment following I.V. administration. (Campbell et al., 2002; Campbell, 2006; DeSantis et al., 2014; Pasenkiewicz-Gierula et al., 2000). Regardless of the cell type from which the CLENs were derived the CLENs were relatively non-toxic to target cells. This is not surprising given the similar composition profile between the target cell membrane and the nano delivery system.

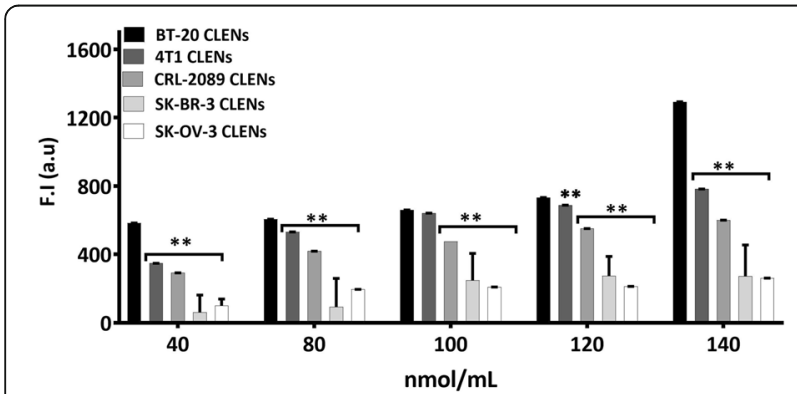

Fig. 3 BT-20 cellular uptake studies. An amount of $1 \times 10^{4}$ BT-20 cells were seeded in a 48 well plate. After $24 \mathrm{~h}$, cells were exposed to different concentrations of fluorescently labeled CLENs for $24 \mathrm{~h}$. The data show cellular uptake of CLENs by all the cells, with the highest degree of uptake observed with BT-20 cells, and the least amount of cellular uptake was observed with the (negative control) ovarian cancer cell line, SK-OV-3. Data are presented as mean \pm S.D.; $(n=6)$

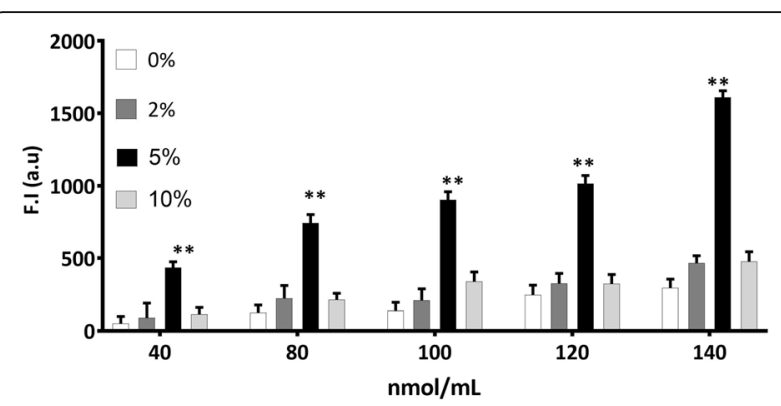

Fig. 4 Effect of cholesterol and PEG inclusion on cellular uptake of CLENs. Four different preparations of 4 T1 CLENs containing 25 mol\% of cholesterol and different mol\% of DPPE-PEG-5000 $(0,2,5$ and 10\%) were evaluated for cellular uptake against $4 \mathrm{~T} 1$ cell line. Rhodamine-labeled CLENs were used for this study. The cells were seeded in a 48-well plate followed by the addition of CLENs for $24 \mathrm{~h}$. The fluorescence intensity used here as an indicator of cell uptake was measured using a fluorescence microplate reader. CLENs containing 5 mol\% of DPPE-PEG-5000 demonstrated the most significant uptake compared to other varieties within the concentration range employed. Data are presented as mean \pm S.D.; $(n=6)$

In general, the greatest degree of selectivity was observed when the breast cancer (target) cells were exposed to CLENs prepared from lipids derived from the target cell. When the target cell population was exposed to lipid material from a different source the results were less impressive, suggesting the mechanism(s) underlying cellular uptake is a function of the lipid composition profile of CLENs, which is cell line-dependent (Figs. 2 and 3).

The incorporation of chemotherapeutic agents in liposomes has been shown to enhance the therapeutic index of incorporated drug agents, either by increasing the drug concentration in tumor cells, or by decreasing exposure to normal healthy tissues (Sharma et al., 2006; Vemuri \& Rhodes, 1995; Pasenkiewicz-Gierula et al., 2000).

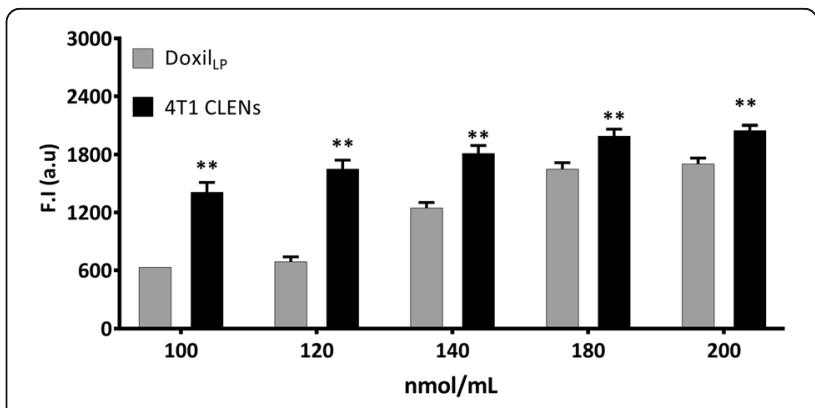

Fig. 5 Comparing cellular uptake of CLENs and Doxil LP. An amount of $1 \times 10^{4} 4 \mathrm{~T} 1$ cells were seeded in a 48 - well plate. After $24 \mathrm{~h}$, cells were incubated for an additional $24 \mathrm{~h}$ with various concentrations of rhodamine labeled CLENs (containing 5 mol\% of PEG-5000) and DoxilLP. The data show that CLENs containing 5 mol\% of DPPE-PEG5000 were taken up to a greater extent compared to the conventional Doxil Lp. Error bars indicate mean \pm S.D. $(n=6)$ 


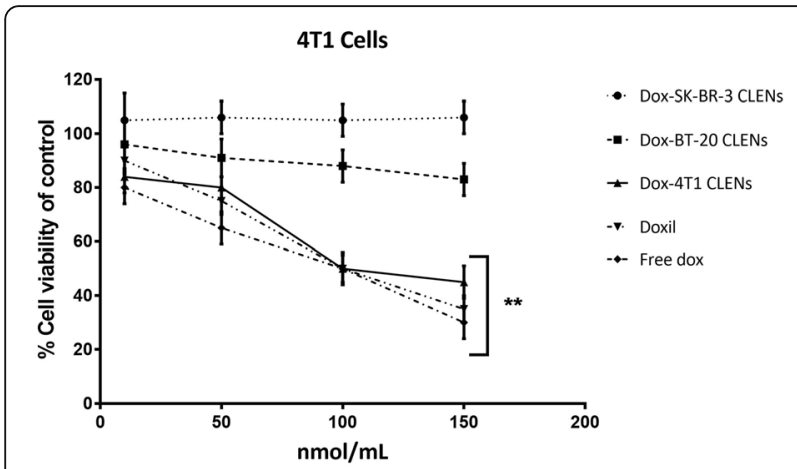

Fig. 6 Cytotoxicity of doxorubicin-loaded CLENs. 4 T1 cells were seeded at $1 \times 10^{4}$ per $\mathrm{ml}$ in a 48-well plate. Cells were exposed to different concentrations of doxorubicin-loaded CLENs (4 T1, BT-20, and SKBR-3). SRB assay was used to determine percent of cell viability $24 \mathrm{~h}$ following exposure to doxorubicin ( $5 \mathrm{~mol} \%$ ). Error bars indicate mean \pm S.D. $(n=6)$

Cholesterol and DPPE-PEG-5000 are commonly used to optimize nanoliposome formulations (Sadzuka et al., 2003; Hatakeyama et al., 2013; Pasenkiewicz-Gierula et al., 2000). Cholesterol is known to increase the packing order and the rigidity of liposomes. The decrease in the permeability of the lipid bilayer due to the inclusion of cholesterol has been shown to improve drug retention for various lipid-based nano-sized drug delivery systems. (Raffy \& Teissié, 1999; Tseng et al., 2007; Sadzuka et al., 2006). PEGylation has offered opportunities to formulate drug carrier molecules that can evade opsonization and relatively rapid blood clearance (Sadzuka et al., 2003; Pasenkiewicz-Gierula et al., 2000; Drummond et al., 1999). In our study, the inclusion of 5 mol\% DPPE-PEG-5000 in CLENs improved cellular uptake over other ratios evaluated ( $5 \mathrm{~mol} \%>0,2$ and $10 \mathrm{~mol} \%$ DPPE-PEG-5000). The experimental finding is particularly noteworthy, suggesting a quite different role of PEG in CLENs compared to more conventional stealth liposomes. CLENs varied in the amount of drug incorporated. The most significant increase in incorporated drug was observed following the inclusion of $25 \mathrm{~mol} \%$ cholesterol ( $86 \%$ drug loaded). The percent cholesterol is greater than that typically used to prepare conventional liposomes (Raffy \& Teissié, 1999; Ohvo-Rekila et al., 2002; Cui et al., 2014). Approximately $44 \%$ of drug was incorporated in CLENs consisting of $50 \mathrm{~mol} \%$ cholesterol, the lowest percent of drug incorporated compared to others evaluated (Table 3; 25>10>0> $50 \mathrm{~mol} \%$ ). Previously published reports support a corelationship between relatively high cholesterol content and liposome instability (Tseng et al., 2007; Decker et al., 2012; Pedrosa et al., 2015). The optimal ratio of cholesterol with respect to conventional components employed is necessary; the balanced optimization will prevent premature drug release and formulation instability.

The cellular uptake of CLENs by the target cells was greater overall when compared to Doxil ${ }_{\mathrm{LP}}$. Moreover, $4 \mathrm{~T} 1$ doxorubicin loaded-CLENs demonstrated more significant growth inhibitory effects. Data also support greater activity against intended target cells compared to non-specific target (control) cell populations. A greater degree of selectivity was observed when both the lipid extracts used to prepare CLENs and the target cell population were derived from the same organ tissue environment (i.e. breast). This result was highly consistent and reproducible. However, the most desirable results were achieved when CLENs were prepared directly from the target cell, when compared to target cells that shared the organ tissue environment only (Figs. 2 and 3).

Not yet identified are the key determinants of selective targeting, as well the driving forces underlying cellular uptake of CLENs. Preliminary evaluation of the entire chloroform-soluble (lipid) fraction of $4 \mathrm{~T} 1$ cells by LC/ MS revealed a variety of glycosphingolipids among other lipid classes (manuscript in preparation). The exact mechanism for why the complex lipid mixture and

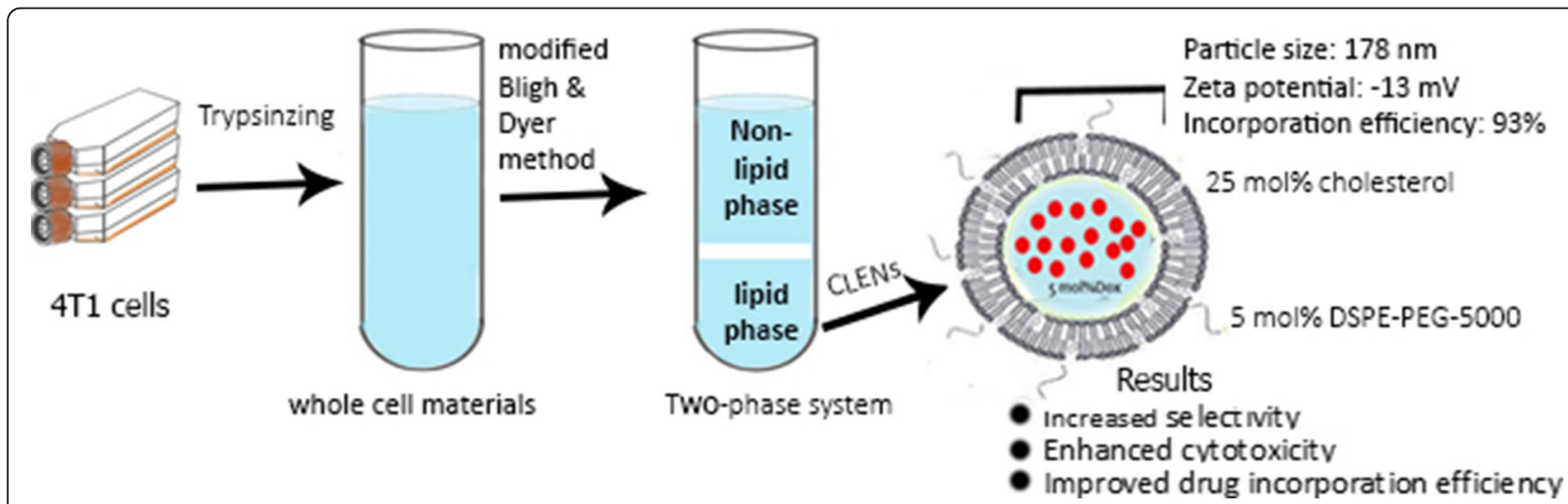

Fig. 7 Isolation of lipid extracts from target cells in preparation of CLENs. The schematic shows the process for preparing CLENs from isolation of the chloroform-soluble fraction which corresponds to the lipid extract phase mixture. The inclusion of cholesterol and DPPE-PEG 5000 in CLENs improved drug incorporation, stability, cellular uptake, and cytotoxicity among other formulation properties 
unique lipid ratio profile of CLENs preferentially accumulate in target cells is not known. Of interest, following evaluation of a different nanosystem composition, Pedrosa and colleagues showed that relatively short-chained glycosphingolipids (SC-GSLs) selectively entered target cell membranes when cells were exposed to SC-GSLs-modified liposomes. The authors suggested that the inclusion of SC-GSLs facilitated the transport of amphiphilic drugs (such as doxorubicin-hydrochloride) compared to nonglycosphingolipid-rich liposomes (36). Our preliminary LC/ MS studies confirm the presence of SC-GSLs in our lipid extracts for $4 \mathrm{~T} 1$, and in extracts derived from other organ tissues as well (data not shown).

\section{Conclusions}

CLENs represent a novel nanoliposome drug platform capable of recognizing target cells with relatively high efficiency compared to more conventional nano-systems. The drug carrier was relatively non-toxic to cells when used at concentrations traditionally used to evaluate nanoparticles in vitro. Our studies collectively support the use of cellular membrane lipid extracts in combination with more conventional components of drug delivery systems to achieve more selective drug targeting. Overall, CLENs were most efficient when applied against intended target cell populations. However, the organ tissue environment appears to play a role in mechanism(s) underlying cell uptake. The results could thus vary depending on the host tissue environment. For this reason, subsequent studies should investigate the relationship between CLENs and the tissue environment.

\section{Abbreviations \\ Chol: Cholesterol; CLENs: Cell membrane lipid extracted nanoliposomes; DOPC: 1,2-Dioleoyl-sn-Glycero-3-Phosphocholine; DOPG: 1, 2-dioleoyl-Sn- glycero-3-[phospho-rac-(1-glycerol)]; Doxil: Stealth liposomal doxorubicin; Doxil P: Doxil lipid preparation only (no drug loaded); DPPE-PEG_5000: 1,2- dipalmitoryl-sn-glycero-3- [phospho-ethanolamine (PEG)]-5000; Rhodamine DPPE: 1,2-dipalmitoyl-sn-glycero-3-phosphoethanolamine-N-(Lissamine rhodamine B sulfonyl); SC-GSLs: Short-chained glycosphingolipids; SRB: Sulforhodamine B; TCA: Trichloroacidic acid}

\section{Acknowledgements}

We thank the Saudi Arabian Culture Mission and Umm Al-Qura University for host institution sponsorship and support for H.A.

\section{Funding}

The research study was funded in part by MCPHS University, and the National Science Foundation CMMI Grant No. 1232339 for corresponding author R.B.C.

\section{Authors' contributions}

This manuscript represents work submitted in partial fulfillment of the Doctoral Degree at MCPHS University for first author (HA), under the direction of (Dr. RBC). HA participated in the experimental design, manuscript draft and performed the experiments. Supervisor for project RBC conceived of the study, participated in the design, coordination and drafts of the manuscript. Both authors read and approved the final manuscript.

\section{Competing interests}

The authors declare that they have no competing interests.

\section{Publisher's Note}

Springer Nature remains neutral with regard to jurisdictional claims in published maps and institutional affiliations.

Received: 24 April 2018 Accepted: 8 July 2018

Published online: 23 July 2018

\section{References}

Allen TM, Hansen C, Martin F, Redemann C, Yau-Young A (1991) Liposomes containing synthetic lipid derivatives of poly(ethylene glycol) show prolonged circulation half-lives in vivo. Biochem Biophys Acta 1066(1):29-36

Bligh EG, Dyer WJ (1959) A rapid method of total lipid extraction and purification. Can J Biochem Phys 37(8):911-917

Campbell RB (2006) Tumor physiology and delivery of nanopharmaceuticals. Anti Cancer Agents Med Chem 6(6):503-512

Campbell RB, Fukumura D, Brown EB, Mazzola LM, Izumi Y et al (2002) Cationic charge determines the distribution of liposomes between the vascular and extravascular compartments of tumors. Cancer Res 62(23):6831-6836

Cui ZK, Edwards K, Orellana AN, Bastiat G, Benoit JP, Lafleur M (2014) Impact of interfacial cholesterol-anchored polyethylene glycol on sterol-rich nonphospholipid liposomes. J Colloid Inter Sci 428:111-120

Dabbas S, Kaushik RR, Dandamudi S, Kuesters GM, Campbell RB (2008) Importance of the liposomal cationic lipid content and type in tumor vascular targeting: physicochemical characterization and in vitro studies using human primany and transformed endothelial cells. Endothelium 15(4):189-201

Dan N (2002) Effect of liposome charge and PEG polymer layer thickness on cell-liposome electrostatic interactions. Biochim Biophys Acta 1564(2):343-348

Dandamudi S, Campbell RB (2007) The drug loading, cytotoxicty and tumor vascular targeting characteristics of magnetite in magnetic drug targeting. Biomaterials 28(31):4673-4683

Decker C, Fahr A, Kuntsche J, May S (2012) Selective partitioning of cholesterol and a model drug into liposomes of varying size. Chem Phys Lipids 165(5):520-529

DeSantis C, Ma J, Bryan L, Jemal A (2014) Breast cancer statistics, 2013. CA Cancer J Clin 64(1):52-62

Deshpande PP, Biswas S, Torchilin VP (2013) Current trends in the use of liposomes for tumor targeting. Nanomedicine (Lond) 8(9):1509-1528

Drummond DC, Meyer O, Hong K, Kirpotin DB, Papahadjopoulos D (1999) Optimizing liposomes for delivery of chemotherapeutic agents to solid tumors. Pharmacol Rev 51(4):691-743

Eloy JO, Claro de Souza M, Petrilli R, Barcellos JP, Lee RJ et al (2014) Liposomes as carriers of hydrophilic small molecule drugs: strategies to enhance encapsulation and delivery. Colloids Surf B Biointerfaces 123c:345-363

Hassan MS, Ansari J, Spooner D, Hussain SA (2010) Chemotherapy for breast cancer (review). Onco Rep 24(5):1121-1131

Hatakeyama H, Akita H, Harashima H (2013) The polyethyleneglycol dilemma: advantage and disadvantage of PEGylation of liposomes for systemic genes and nucleic acids delivery to tumors. Biol Pharm Bull 36(6):892-899

Jain A, Jain SK (2008) PEGylation: an approach for drug delivery. A review. Crit Rev Ther Drug Carrier Syst 25(5):403-447

Jemal A, Bray F, Center MM, Ferlay J, Ward E, Forman D (2011) Global cancer statistics. C A 61 (2):69-90

Kalra AV, Campbell RB (2006) Development of 5-FU and doxorubicin-loaded cationic liposomes against human pancreatic cancer: implications for tumor vascular targeting. Pharm Res 23(12):2809-2817

Lu Y, Low PS (2002) Folate-mediated delivery of macromolecular anticancer therapeutic agents. Adv Drug Deliv Rev 54(5):675-693

Mathias CJ, Wang S, Waters DJ, Turek JJ, Low PS et al (1998) Indium-111-DTPAfolate as a potential folate-receptor-targeted radiopharmaceutical. J Nuc Med 39(9):1579-1585

Moulder S, Hortobagyi GN (2008) Advances in the treatment of breast cancer. Clin pharm thera 83(1):26-36

Ohvo-Rekila H, Ramstedt B, Leppimaki P, Slotte JP (2002) Cholesterol interactions with phospholipids in membranes. Prog Lipid Res 41(1):66-97

Pasenkiewicz-Gierula M, Rog T, Kitamura K, Kusumi A (2000) Cholesterol effects on the phosphatidylcholine bilayer polar region: a molecular simulation study. Biophys J 78(3):1376-1389

Pedrosa LR, Ten Hagen TL, Suss R, van Hell A, Eggermont AM, Verheij M et al (2015) Short-chain glycoceramides promote intracellular mitoxantrone delivery from novel nanoliposomes into breast cancer cells. Pharma res 32(4):1354-1367 
Raffy S, Teissié J (1999) Control of lipid membrane stability by cholesterol content. Biophys J 76(4):2072-2080

Rivenbark AG, O'Connor SM, Coleman WB (2013) Molecular and cellular heterogeneity in breast cancer: challenges for personalized medicine. Am J Path 183(4):1113-1124

Ryan SM, Mantovani G, Wang X, Haddleton DM, Brayden DJ (2008) Advances in PEGylation of important biotech molecules: delivery aspects. Expert Opin Drug Deliv 5(4):371-383

Sadzuka Y, Kishi K, Hirota S, Sonobe T (2003) Effect of polyethyleneglycol (PEG) chain on cell uptake of PEG-modified liposomes. J Liposome Res 13(2):157-172

Sadzuka Y, Sugiyama I, Tsuruda T, Sonobe T (2006) Characterization and cytotoxicity of mixed polyethyleneglycol modified liposomes containing doxorubicin. Int J Pharm 312(1-2):83-89

Sharma G, Anabousi S, Ehrhardt C, Ravi Kumar MN (2006) Liposomes as targeted drug delivery systems in the treatment of breast cancer. J Drug Deliv 14(5):301-310

Taylor KMG, Taylor G, Kellaway IW, Stevens J (1990) Drug entrapment and release from multilamellar and reverse-phase evaporation liposomes. Int J Pharm 58(1):49-55

Torchilin VP (2005) Recent advances with liposomes as pharmaceutical carriers. Nat Rev Drug Discov 4(2):145-160

Tseng LP, Liang HJ, Chung TW, Huang YY, Liu DZ (2007) Liposomes incorporated with cholesterol for drug release triggered by magnetic field. J Med Bio Eng 27(1):29-34

Vemuri S, Rhodes CT (1995) Preparation and characterization of liposomes as therapeutic delivery systems: a review. Pharm Acta Helv 70(2):95-111

Verma S, Dent S, Chow BJ, Rayson D, Safra T (2008) Metastatic breast cancer: the role of pegylated liposomal doxorubicin after conventional anthracyclines. Can Treat Rev 34(5):391-406

\section{Submit your manuscript to a SpringerOpen ${ }^{\circ}$ journal and benefit from:}

- Convenient online submission

- Rigorous peer review

- Open access: articles freely available online

High visibility within the field

- Retaining the copyright to your article

Submit your next manuscript at $\boldsymbol{\nabla}$ springeropen.com 